\title{
Risk of colitis in immune checkpoint inhibitors and in chemotherapy/placebo for solid tumors: a systematic review and meta-analysis
}

\author{
Wei Wei ${ }^{1}$ Yun Ding ${ }^{2}$, Jiajia $\mathrm{He}^{1}$, Jun $\mathrm{Wu}^{1}$ \\ ${ }^{1}$ Department of Oncology, ${ }^{2}$ Department of Radiotherapy, The Third Affiliated Hospital of Soochow University, Changzhou, China \\ Contributions: (I) Conception and design: W Wei; (II) Administrative support: J Wu; (III) Provision of study materials or patients: W Wei; (IV) \\ Collection and assembly of data: Y Ding, J He; (V) Data analysis and interpretation: W Wei; (VI) Manuscript writing: All authors; (VII) Final \\ approval of manuscript: All authors. \\ Correspondence to: Jun Wu. Department of Oncology, The Third Affiliated Hospital of Soochow University, 185 Juqian Street, Changzhou, China. \\ Email:wujun68@sina.com.
}

Background: We conducted a systematic review and meta-analysis in an attempt to evaluate the risk of colitis in immune checkpoint inhibitors (ICIs) and in chemotherapy/placebo for solid tumors.

Methods: Embase and PubMed were searched with specific keywords of our concern. Collected data were processed using Stata 14.0 and Review Manager 5.3. Odds ratios (ORs) of grade 1-5 and grade 3-5 colitis in treatment arms compared with control arms were pooled.

Results: A total of 11,226 patients from 20 phase 2/3 randomized controlled trials were included into this meta-analysis. Treatment strategies involving ICIs resulted in a significant increased risk of any grade colitis compared with chemotherapy/placebo (grade 1-5 colitis OR: 8.78, 95\% CI: 5.88-13.12, P<0.001; grade 3-5 colitis OR: 10.82, 95\% CI: $6.02-19.44, \mathrm{P}<0.001)$. ICIs plus chemotherapy showed higher risk of colitis than placebo plus chemotherapy (grade $1-5$ colitis OR: 12.85 , 95\% CI: 4.98-33.18, $\mathrm{P}<0.001$; grade $3-5$ colitis OR: 9.17, 95\% CI: 3.31-25.41, $\mathrm{P}<0.001$ ). ICIs monotherapy showed higher risk of colitis than placebo (grade 1-5 colitis OR: 11.98, 95\% CI: 6.789-21.13, P<0.001; grade 3-5 colitis OR: 21.46, 95\% CI: 7.80-59.04, $\mathrm{P}<0.001$ ). When comparing risk of colitis among three ICIs, results suggested that CTLA-4 antibodies (grade 1-5 colitis OR: 13.43, 95\% CI: 7.88-22.89, $\mathrm{P}<0.001$; grade 3-5 colitis OR: 15.74, 95\% CI: 7.34-33.78, $\mathrm{P}<0.001$ ) tended to generate the highest risk of colitis and PD-1 antibodies (grade 1-5 colitis OR: 3.64, 95\% CI: 1.87-7.06, $\mathrm{P}<0.001$; grade 3-5 colitis OR: 4.56, 95\% CI: 1.68-12.36, $\mathrm{P}=0.003)$ the lowest. Subgroup analyses between different tumor types showed that non-small-cell lung cancer (NSCLC) patients receiving ICIs treatment seemed to have the lowest risk of colitis (grade 1-5 colitis OR: 6.41, 95\% CI: 2.26-18.21, $\mathrm{P}<0.001$; grade 3-5 colitis OR: 5.64, 95\% CI: 1.69-18.87, $\mathrm{P}=0.005)$. Publication bias was present in this study, but trim and fill analysis was done and confirmed the results were reliable.

Conclusions: Among different treatment strategies for solid tumors, combination of ICIs and chemotherapy produces higher risk of colitis than ICIs monotherapy. CTLA-4 antibodies tended to generate highest risk of colitis, and PD-1 antibodies the lowest. Compared with melanoma and some other solid tumors, NSCLC patients receiving ICIs treatment were least likely to develop colitis of any grade.

Keywords: Colitis; cancer; immune therapy

Submitted Dec 19, 2019. Accepted for publication May 28, 2020.

doi: $10.21037 /$ tcr-19-2872

View this article at: http://dx.doi.org/10.21037/tcr-19-2872 


\section{Introduction}

The oncology field of the $21^{\text {st }}$ century witnessed the groundbreaking entrance of cancer immune therapy. Immune checkpoint inhibitors (ICIs) targeting programmed cell death 1 (PD-1), programmed cell death ligand 1 (PDL1) and cytotoxic T lymphocyte-associated protein 4 (CTLA-4) are able to reverse the immune-suppressed tumor microenvironment and boost patient antitumor immune activity (1-3). Numerous randomized, controlled trials involving ICIs have reported inspiring efficacy and safety results, leading to FDA approvals of various ICIs for clinical use. By far, ICIs have literally reshaped the treatment and prognosis of many solid tumors.

Providing that immune checkpoint molecules, functioning as immune "brakes" in a healthy host, are responsible for maintaining organ self-tolerance and containing inflammatory damage, it is only logical to expect certain side effects from ICIs. Presumably as a result of immune over-stimulation in certain parts of the human body, ICIs are known to cause specific adverse effects, such as hypothyroidism, immune related pneumonitis and immune related myositis (4-7). As more cancer patients begin to gain survival benefits from ICIs in clinical practice, ICIs related adverse event reports are gradually drawing attention. Among the most common adverse events are colitis, pneumonitis, rash or pruritus, liver toxicity and nephritis (4). Some serious adverse events, such as immune related myocarditis and encephalitis could be fatal if not recognized dealt with in early stages $(8,9)$.

Immune related colitis, one of the adverse events reported in multiple randomized controlled trials, is a common side effect in patients receiving ICIs in clinical practice. In this meta-analysis we choose to focus on the risk of immune related colitis in cancer patients, mainly because we have observed several cases of this particular adverse event during our daily work as physicians. Some patients were regrettably delayed or even forced to withdraw from an ICIs involved treatment that was substantially benefitting them, thus we strive to explore the risk of immune related colitis in cancer patients receiving ICIs treatment in the hope of providing a safer treating strategy to different patients. Currently, eight ICIs have been approved by FDA, including three PD-1 inhibitors (nivolumab, pembrolizumab and cemiplimab), three PD-L1 inhibitors (atezolizumab, durvalumab and avelumab), and two CTLA4 inhibitors (ipilimumab and tremelimumab). This metaanalysis focused on the risk of colitis in solid tumor patients receiving these drugs in comparison with chemotherapy or placebo. We present the following article in accordance with the PRISMA reporting checklist (available at http:// dx.doi.org/10.21037/tcr-19-2872).

\section{Methods}

\section{Search strategy}

We searched through PubMed and EMBASE to screen clinical trials involving ICIs from January 1970 to October 2019. The aim of this study was to evaluate the risk of colitis potentially associated with ICIs for solid tumors, thus our search strategy combined text word and MeSH (Emtree for EMBASE database accordingly) with the terms "colitis" and "PD-1" or "PD-L1" or "CTLA-4" or "Pembrolizumab" or "Nivolumab" or "Cemiplimab" or "Atezolizumab" or "Durvalumab" or "Avelumab" or "Ipilimumab" or "Tremelimumab". The combination was adjusted in each database. Only published clinical trials reporting the risk of colitis were included. We also did a manual search upon each study according to its references. The searching and collecting process was in line with the PRISMA 2009 statement.

\section{Criteria for inclusion and exclusion}

The inclusion criteria for eligible studies were as follows: (I) published in English literature; (II) phase 2/3 randomized control trials (RCTs) with primary endpoints including overall survival (OS) or progression-free survival (PFS); (III) histologically diagnosed solid tumors; (IV) treatment arms involving ICIs combined therapy or monotherapy; (V) containing complete data of colitis incidence rate.

The exclusion criteria were as follows: (I) reviews, letters, case reports, ongoing trials, conference abstracts without original data; (II) phase 1 RCTs; (III) studies conducted on animals or cell lines; (IV) studies without a control arm of chemotherapy/placebo; (V) studies reported in other languages.

\section{Quality assessment and data extraction}

Cochrane Handbook for systematic Reviews of Interventions was used to assess the risk of bias for each included study. Two reviewers independently assessed the quality of every study included in this meta-analysis using Review Manager 5.3. Should disagreements occur, a 


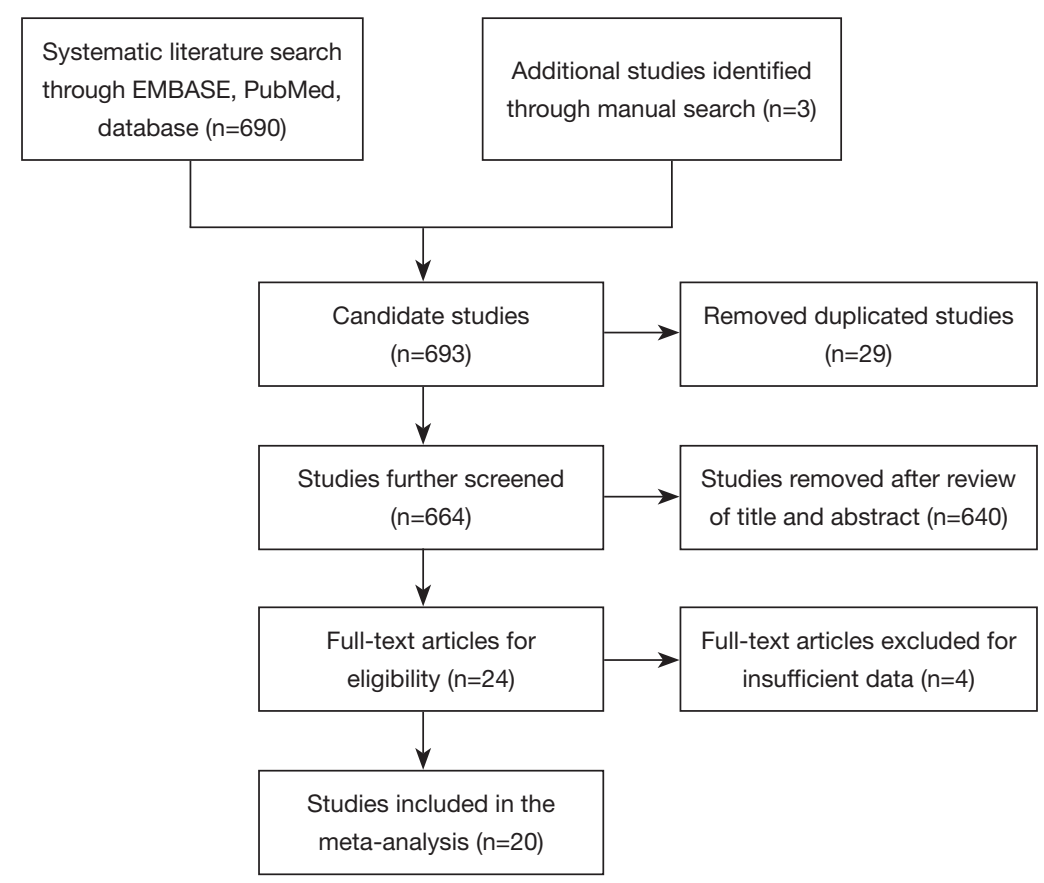

Figure 1 Flowchart of data collection.

third reviewer would join to reach consensus. This metaanalysis was based on previously published high quality clinical trials; thus no ethical approval was required. Ethical approvals of each included study can be found in the original article.

Data extraction was done by two reviewers independently, and the indexes collected were as follows: surname of the first author, year of publication, tumor histology, drugs applied in the intervention group and control group, number of events (any grade and grade 3-5 colitis) in the intervention group and the control group. If data extraction displayed inconsistency between two reviewers, a third reviewer would join to check again.

\section{Statistical analysis}

Quality assessment was done by Review Manager 5.3. All extracted data were processed by Stata 14.0. The odds ratios (ORs) for any grade and grade 3-5 colitis with 95\% CI were pooled. If no significant heterogeneity occurred, fixed effects model was applied. Otherwise, random effects model would be applied. L'abbe plot and Galbraith Radial plot were drawn to evaluate potential heterogeneity. Different subgroup analyses were done to explore influence factors. Sensitivity analysis was done to determine the influence of each study on the pooled result. A funnel plot as well as Egger's and Begg's test was performed to evaluate publication bias. If publication bias existed, trim and fill analysis would be done to check the stability of the pooled results.

\section{Results}

\section{Literature screening and data extraction}

As shown in Figure 1, originally 690 studies were identified via our search strategy, plus 3 studies added by manual search. After removing 29 duplicated studies, we reviewed the title and abstract of 664 studies, leaving 24 studies for further selection. Then we went through the whole text as well as supplementary materials of these potentially eligible studies to remove those with insufficient data. Eventually 20 studies comprising of 11,226 patients were included into this meta-analysis (10-29).

Two investigators independently extracted the following data from the included studies: surname of the first author, year of publication, study type, histology, study endpoint, treatment arms, number of patients in different arms, number of any grade and grade 3-5 colitis in different arms. Disagreements between two reviewers were resolved by a third reviewer. All data involved in this meta-analysis were 
extracted from previously published clinical trials, thus no ethical approval was required.

\section{Study characteristics and quality assessment}

The characteristics of all included studies are listed in Table 1. A total of 11,226 patients from 20 studies were included into this meta-analysis, all of which were phase $2 / 3$ randomized controlled trials. These twenty studies covered melanoma, non-small cell lung cancer (NSCLC), small cell lung cancer (SCLC), prostate cancer, malignant mesothelioma, urothelial carcinoma and head-and-neck squamous cell carcinoma. ICIs involved were PD-1 antibodies (nivolumab and pembrolizumab), PD-L1 antibodies (atezolizumab) and CTLA-4 antibodies (ipilimumab and tremelimumab). Six studies compared ICIs plus chemotherapy with placebo plus chemotherapy. Four studies compared ICIs with placebo. Ten studies compared ICIs with chemotherapy.

Quality assessment was performed according to Cochrane Handbook for systematic Reviews of Intervention via Review Manager 5.3, as shown in Figure 2.

\section{Risk of colitis in different treatment strategies involving ICIs}

We were able to collect 20 studies comparing ICIs treatment (PD-1/PD-L1/CTLA-4 antibodies) and chemotherapy/ placebo, or comparing ICIs plus chemotherapy and placebo plus chemotherapy. Fixed effects model was applied for the absence of significant heterogeneity.

As shown in Figure 3 (A: grade 1-5 colitis, B: grade 3-5 colitis), pooled data suggested that treatment strategies involving ICIs resulted in a significant increased risk of grade $1-5$ as well as grade $3-5$ colitis compared with control arms of placebo or chemotherapy (grade $1-5$ colitis OR: 8.78, 95\% CI: $5.88-13.12, \mathrm{P}<0.001$; grade $3-5$ colitis OR: 10.82, 95\% CI: 6.02-19.44, $\mathrm{P}<0.001)$.

Combined treatment containing both ICIs and chemotherapy also showed higher risk of grade 1-5 and grade 3-5 colitis than the control arm of placebo plus chemotherapy (grade 1-5 colitis OR: 12.85, 95\% CI: 4.98-33.18, $\mathrm{P}<0.001$; grade 3-5 colitis OR: 9.17 , 95\% CI: 3.31-25.41, $\mathrm{P}<0.001)$.

When comparing ICIs monotherapy with placebo, ICIs monotherapy obviously showed higher risk of grade 1-5 and grade 3-5 colitis than placebo (grade 1-5 colitis OR: 11.98, 95\% CI: 6.789-21.13, $\mathrm{P}<0.001$; grade $3-5$ colitis OR:
21.46, 95\% CI: 7.80-59.04, $\mathrm{P}<0.001)$.

Between ICIs monotherapy and chemotherapy, ICIs monotherapy still showed a slightly higher risk of grade 1-5 and grade 3-5 colitis than chemotherapy (grade 1-5 colitis OR: $2.93,95 \%$ CI: $1.38-6.18, \mathrm{P}=0.005$; grade $3-5$ colitis OR: $3.18,95 \%$ CI: $1.07-9.46, \mathrm{P}=0.038)$.

According to the ORs mentioned above, when both compared with ICIs monotherapy, chemotherapy seemed to present higher risk of grade 1-5 and grade 3-5 colitis than placebo. When both compared with chemotherapy, ICIs plus chemotherapy seemed to produce higher risk of immune related colitis than ICIs monotherapy.

\section{Risk of colitis in PD-1, PD-L1 and CTLA-4 antibody treatment}

This meta-analysis included studies using PD-1, PD-L1 and CTLA-4 inhibitors. Subgroup analyses concerning different ICIs were shown in Figure 4 (A: grade 1-5 colitis, B: grade $3-5$ colitis).

Treatment arms involving CTLA-4 antibodies presented higher risk of grade 1-5 and grade 3-5 colitis than control arms (grade 1-5 colitis OR: 13.43, 95\% CI: 7.88-22.89, $\mathrm{P}<0.001$; grade 3-5 colitis OR: 15.74 , 95\% CI: $7.34-$ 33.78, $\mathrm{P}<0.001)$. Similar results were seen in treatment arms involving PD-1 antibodies compared with control arms (grade 1-5 colitis OR: $3.64,95 \%$ CI: $1.87-7.06$, $\mathrm{P}<0.001$; grade $3-5$ colitis OR: 4.56 , 95\% CI: $1.68-12.36$, $\mathrm{P}=0.003)$. Treatment involving $\mathrm{PD}-\mathrm{L} 1$ antibodies also seemingly induced higher risk than control arms, showing no significant differences. However, this meta-analysis only contained two studies concerning PD-L1 antibodies, where Grade 3-5 colitis only occurred in one. Both studies compared PD-L1 inhibitors with chemotherapy.

When comparing risk of colitis among three ICIs, results suggested that CTLA-4 antibodies tended to generate the highest risk of colitis and PD-1 antibodies the lowest.

\section{Risk of colitis in ICIs treatment for different tumor bistology}

Providing that different solid tumors respond to ICIs differently, we conducted a subgroup analysis among different tumor histology, as shown in Figure 5 (A: grade 1-5 colitis, B: grade 3-5 colitis). Studies were categorized into three subgroups: melanoma, NSCLC and other tumors, which included SCLC, prostate cancer, malignant mesothelioma, urothelial carcinoma and head-and-neck 


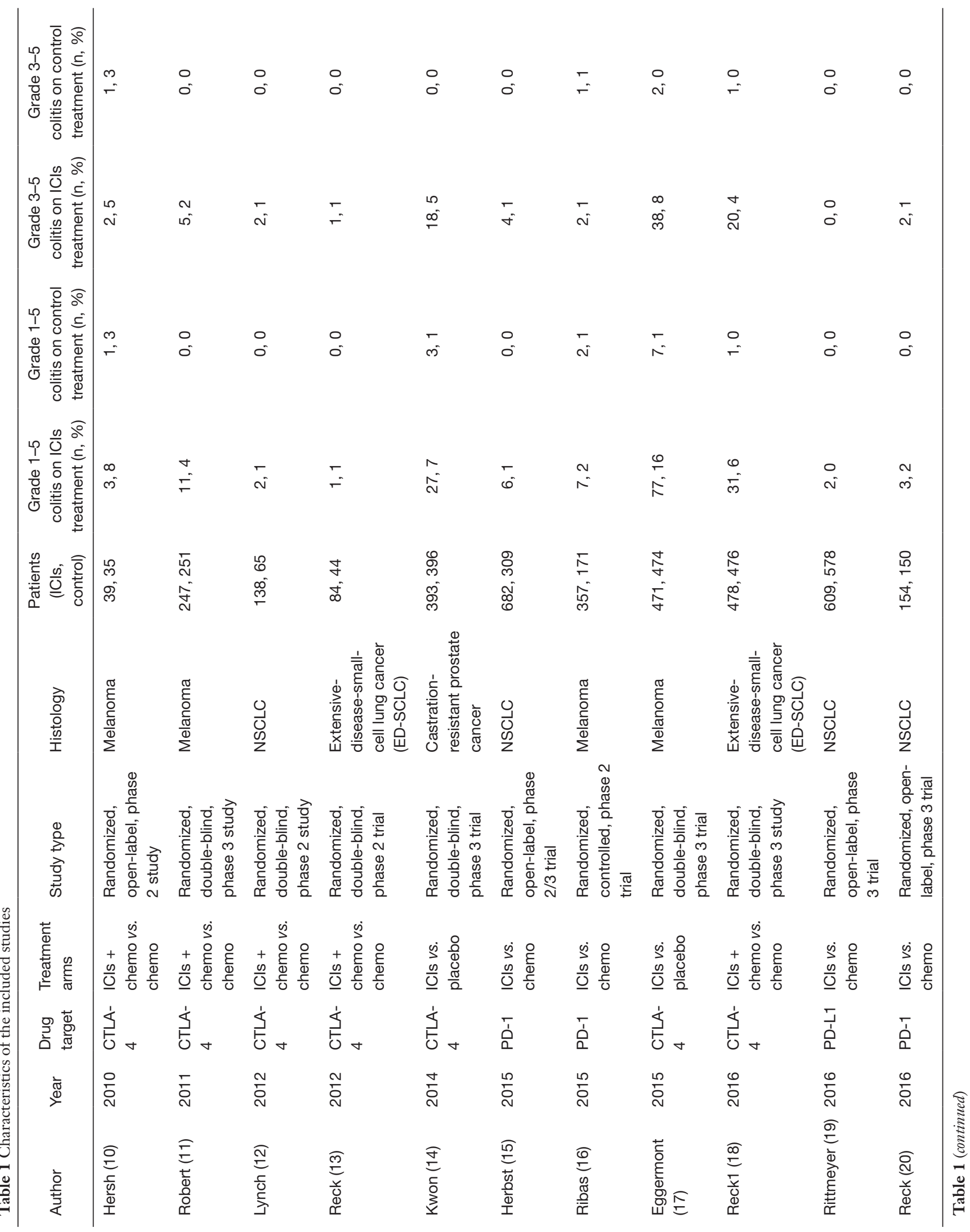




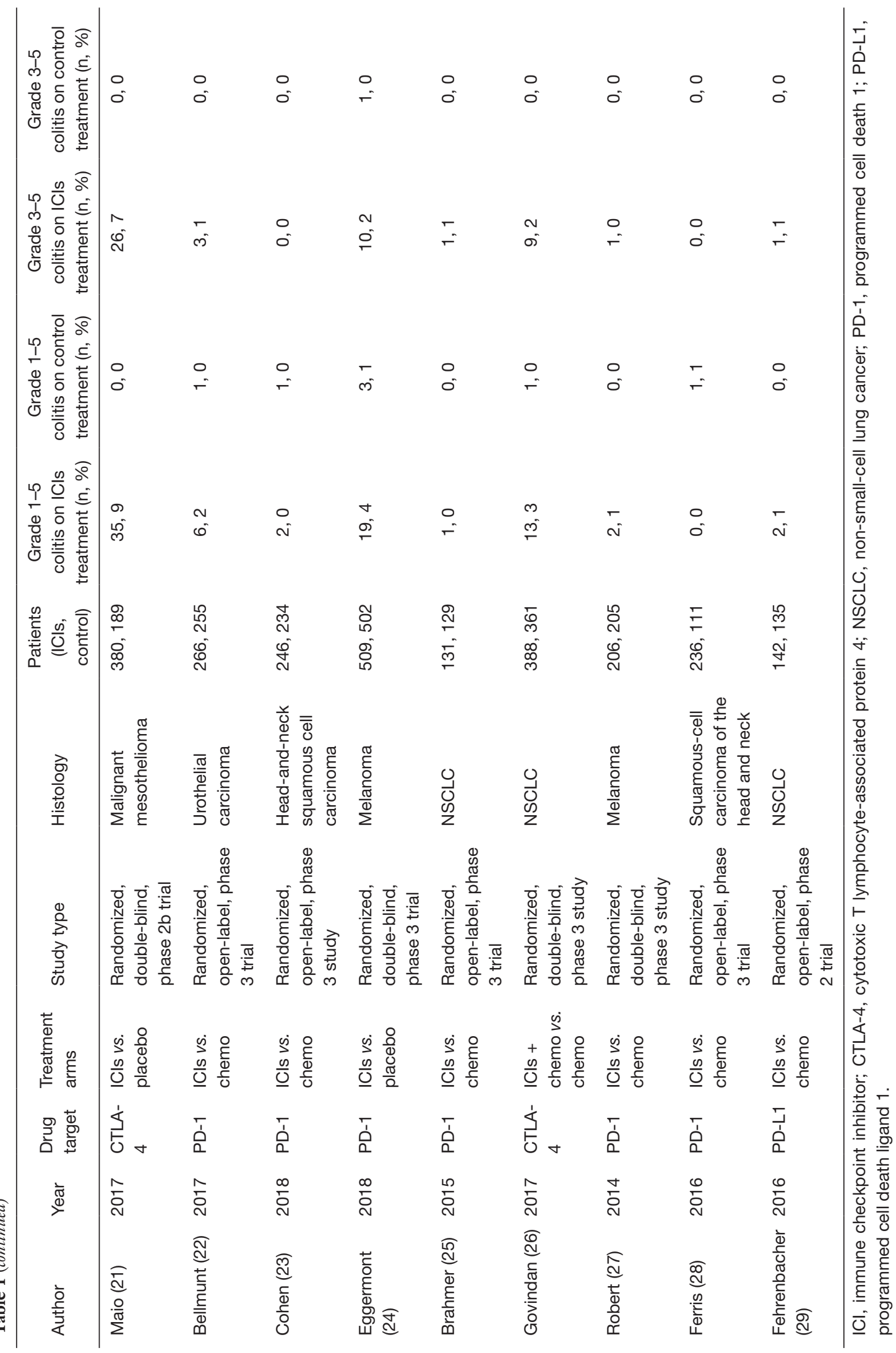




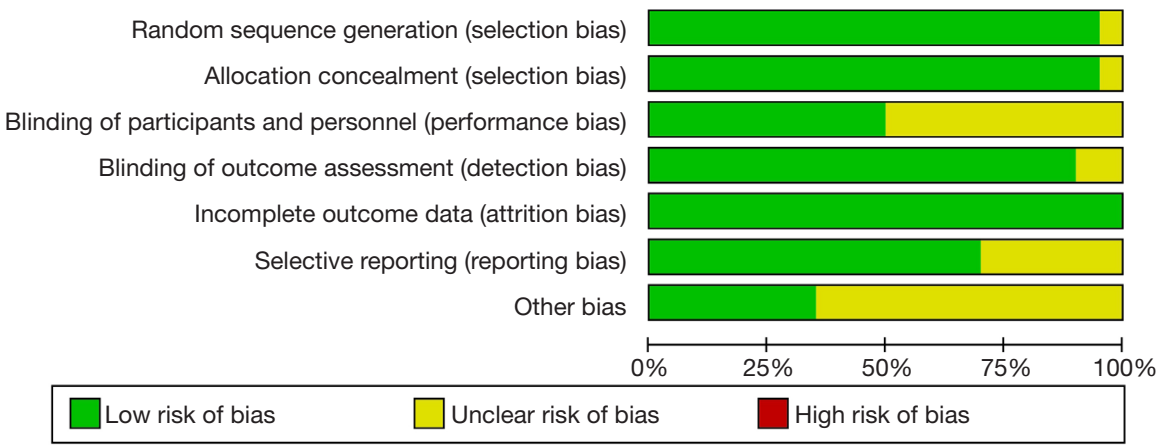

Figure 2 Quality assessment of included studies.

squamous cell carcinoma.

ICIs in melanoma patients showed significant higher risk of colitis (grade 1-5 colitis OR: 8.71, 95\% CI: 5.00-15.17, $\mathrm{P}<0.001$; grade 3-5 colitis OR: 9.43, 95\% CI: 4.11-21.68, $\mathrm{P}<0.001$ ). Similar results were found in NSCLC patients (grade $1-5$ colitis OR: 6.41, 95\% CI: 2.26-18.21, $\mathrm{P}<0.001$; grade $3-5$ colitis OR: 5.64, 95\% CI: $1.69-18.87, \mathrm{P}=0.005$ ) and in other tumors (grade 1-5 colitis OR: $10.04,95 \%$ CI: 4.98-20.21, $\mathrm{P}<0.001$; grade 3-5 colitis OR: 10.82, 95\% CI: 6.02-19.44, $\mathrm{P}<0.001)$.

According to our subgroup analysis, NSCLC patients receiving ICIs treatment seemed to have the lowest risk of colitis, while melanoma patients in ICIs treatment should be more carefully on the watch for colitis events.

\section{Heterogeneity analysis}

No significant overall heterogeneity of grade $1-5 / 3-5$ colitis incidence was found among all the studies included in this meta-analysis (I-squared $=6 \%, \mathrm{P}=0.382$ ). In subgroup analyses, marginal heterogeneity occurred in subgroup of other tumors (I-squared $=50.3 \%, \mathrm{P}=0.061$ ). L'abbe plot and Galbraith Radial plot were drawn to further confirm that no significant heterogeneity existed, as shown in Figure S1 (A: grade 1-5 colitis, B: grade 3-5 colitis) and Figure $S 2$ (A: grade 1-5 colitis, B: grade 3-5 colitis) in the supplementary appendix online.

\section{Publication bias}

We performed Egger's and Begg's test to evaluate potential publication bias among different studies in this meta-analysis, as shown in Figure $S 3$ (A: grade 1-5 colitis, B: grade 3-5 colitis) and Figure S4 (A: grade 1-5 colitis, B: grade 3-5 colitis) in the supplementary appendix online. Both Egger's and Begg's test suggested there was potential publication bias (grade 1-5 colitis:
Egger's test $\mathrm{P}=0.070$, Begg's test $\mathrm{P}=0.015$; grade $3-5$ colitis: Egger's test $\mathrm{P}=0.061$, Begg's test $\mathrm{P}=0.029$ ). Funnel plots were drawn in Figure 6 (A: grade 1-5 colitis, B: grade 3-5 colitis). We further conducted trim and fill analysis. The results, as shown in Figure 7 (A: grade 1-5 colitis, B: grade 3-5 colitis), suggested that the results of this meta-analysis were reliable.

\section{Sensitivity analysis}

We conducted influence analysis via STATA 14.0 to evaluate the influence of each study on the overall result. As shown in Figure S5 (A: grade 1-5 colitis, B: grade 3-5 colitis) in the supplementary appendix online, no single study had significant influence on the overall results.

\section{Discussion}

To our knowledge, this is the first meta-analysis to compare the risk of colitis in ICIs treatment and that in chemotherapy/placebo. After searching through several major databases, we identified 11,226 patients from 20 eligible studies that compared treatment involving ICIs with placebo/chemotherapy. The studies covered various solid tumors including melanoma, NSCLC, SCLC, prostate cancer, malignant mesothelioma, urothelial carcinoma and head-and-neck squamous cell carcinoma. ICIs drugs involved were nivolumab, pembrolizumab, atezolizumab, ipilimumab and tremelimumab. Pooled results displayed a consistent increased risk of grade 1-5 and grade 3-5 colitis from treatment involving ICIs compared with placebo or chemotherapy. Pooled ORs also suggested that CTLA4 antibodies tended to generate the highest risk of colitis and PD-1 antibodies the lowest. NSCLC patients receiving ICIs treatment seemed to have the lowest risk of colitis. No significant heterogeneity was found. Publication bias 
A

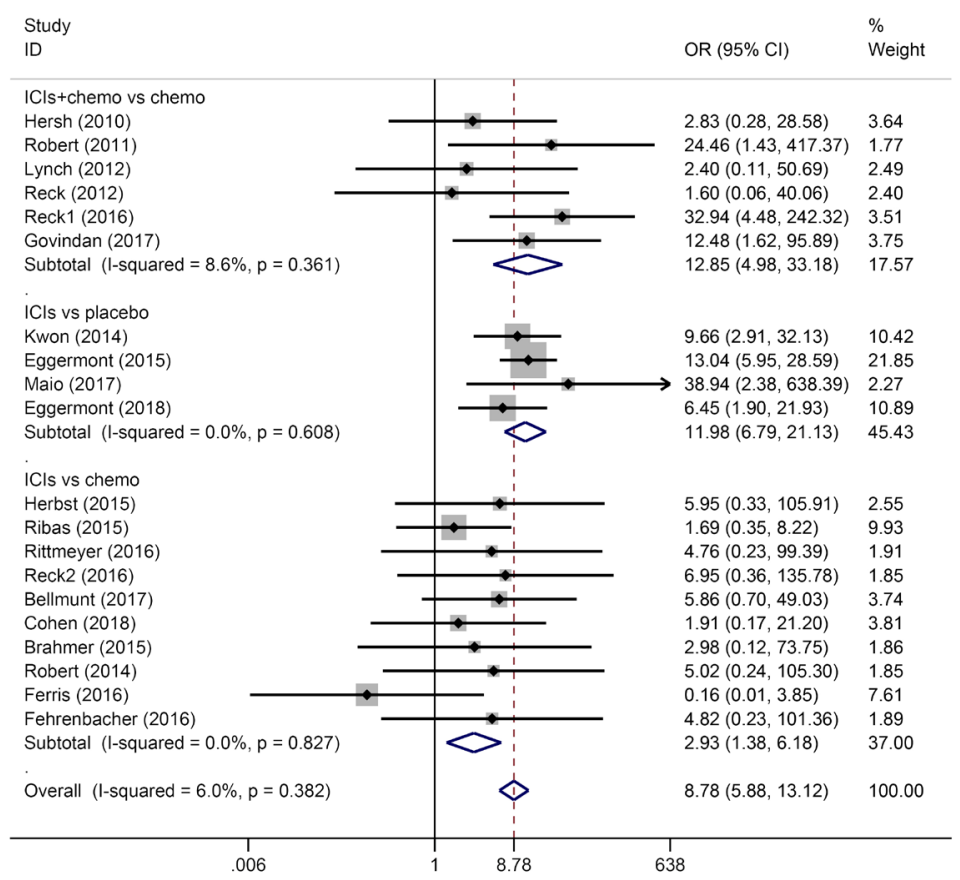

B

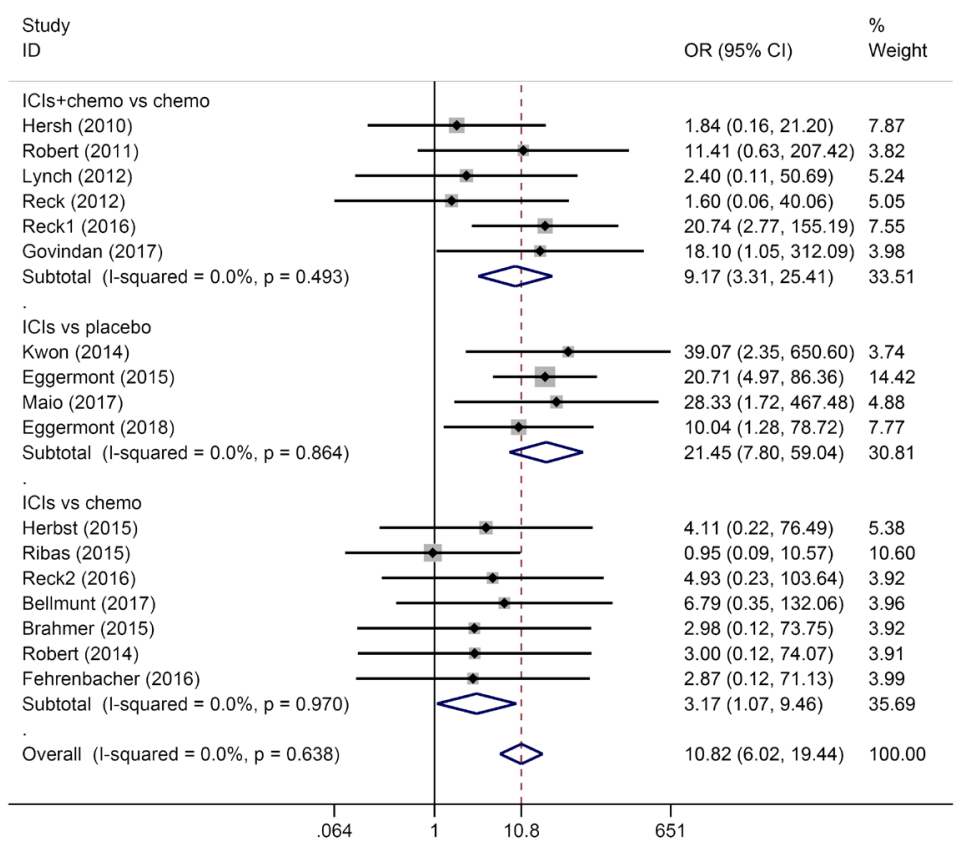

Figure 3 Risk of colitis in different treatment strategies involving ICIs. (A) Grade 1-5 colitis, (B) grade 3-5 colitis. ICI, immune checkpoint inhibitor.

existed, but both trim and fill analysis and sensitivity analysis confirmed that the pooled results were reliable.

ICIs originally made their big entrance in late-stage melanoma patients, CTLA-4 antibodies being the first and soon followed by PD-1/PD-L1 antibodies. ICIs showed tremendous efficacy in these patients and sparkled a trail of clinical trials in other tumors. As numerous clinical trials carry on, inspiring results continue to support 
A
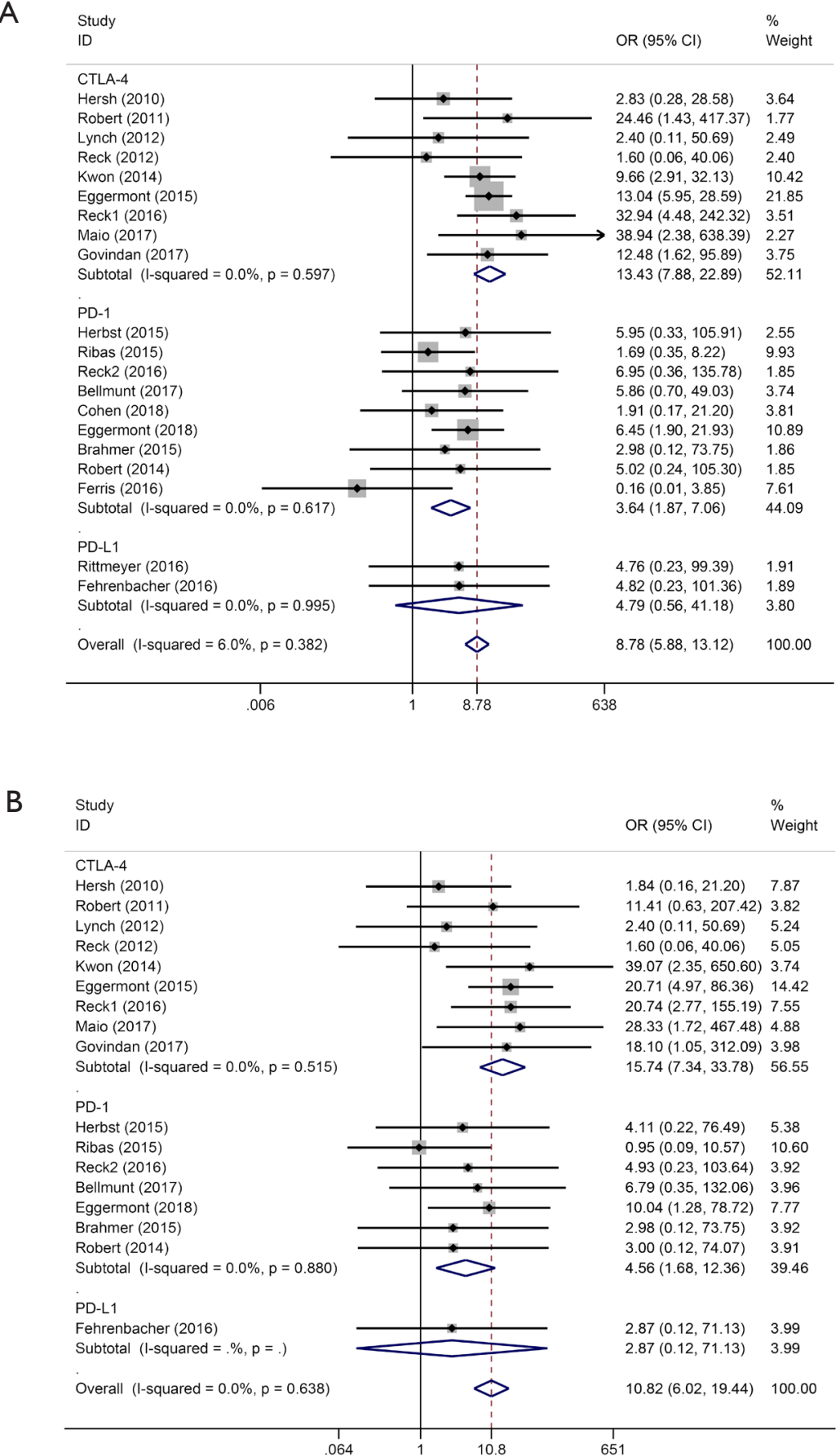

Figure 4 Risk of colitis in PD-1, PD-L1 and CTLA-4 antibody treatment. (A) Grade 1-5 colitis, (B) grade 3-5 colitis. PD-1, programmed cell death 1; PD-L1, programmed cell death ligand 1; CTLA-4, cytotoxic T lymphocyte-associated protein 4.

the efficacy of immune therapy in various tumors. Up until 2019, indications for ICIs have covered melanoma, NSCLC, SCLC, head-and-neck cancer, classic Hodgkin's lymphoma, renal cell carcinoma, urothelial carcinoma, gastric/gastroesophageal adenocarcinoma, hepatocellular carcinoma, colorectal carcinoma, etc., all with certain specific conditions. Bringing significant survival benefits and relatively mild side effects compared with chemotherapy, 
A

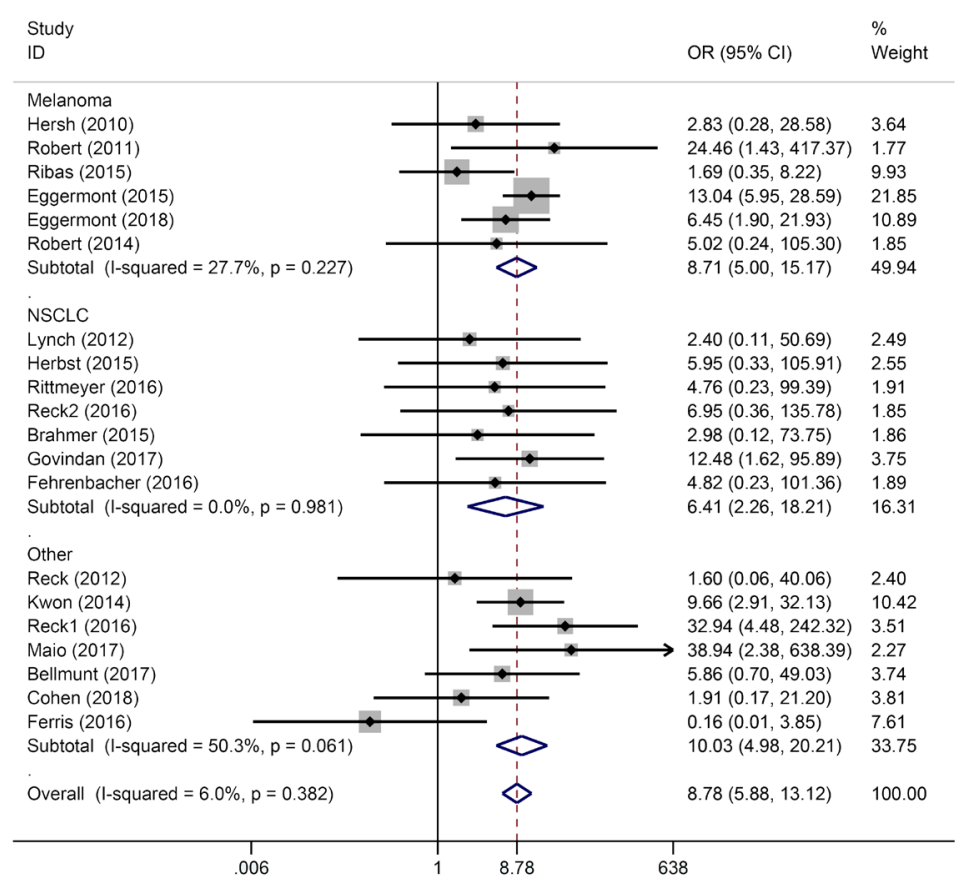

B

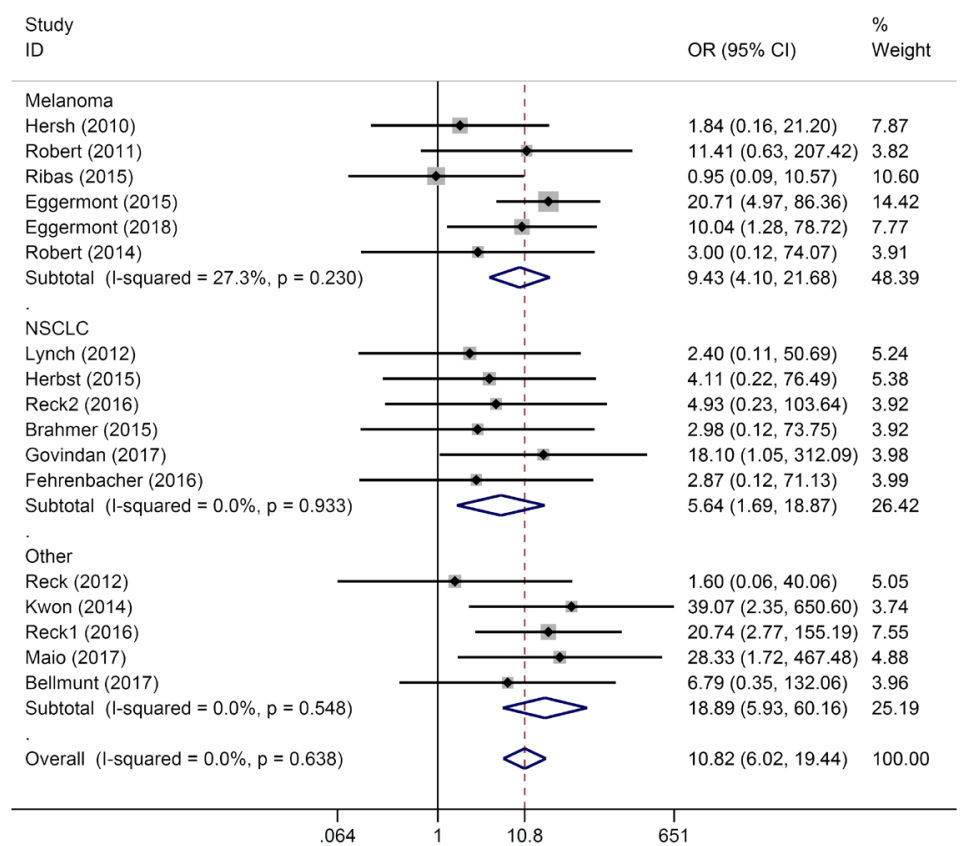

Figure 5 Risk of colitis in ICIs treatment for different tumor histology. (A) Grade 1-5 colitis, (B) grade 3-5 colitis. ICI, immune checkpoint inhibitor.

immune therapy is moving towards the frontline of the battle against cancer, taking up a major role instead of holding the last defense. For those who are denied the chance of targeted therapy and left with the only choice of chemotherapy or radiotherapy, ICIs may very well set them on a brand new path to a better outcome. 

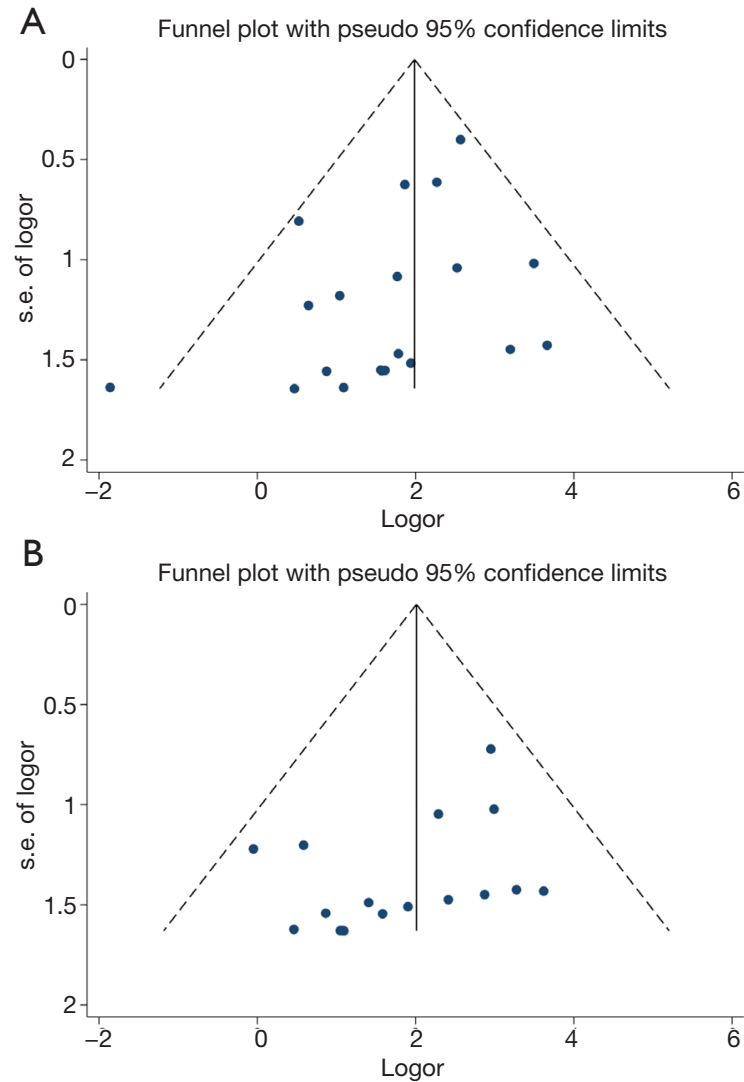

Figure 6 Funnel plots for publication bias. (A) Grade 1-5 colitis, (B) grade 3-5 colitis.

As immune therapy came under the spotlight these years, considerable incidence rates of various immune related adverse events begin to draw attention. In the process of reviving a patient's antitumor response, ICIs inhibit certain immune brakes and release an active, sometimes over-active immune system to fight against tumor cells in the patient's body, bringing collateral damage. Colitis, among other adverse events, is rather frequently reported in multiple randomized clinical trials. A patient with ICIs induced colitis is likely to present symptoms of abdominal pain and diarrhea with or without bloody purulent stool. Endoscopic exams tend to show diffuse ulceration and edema and biopsy samples reveal a lymphocytic and neutrophilic infiltration with apoptotic mucosal epithelial cells and crypt abscesses (4).

As is mentioned above, we attempt to compare the risk of colitis from ICIs and from chemotherapy/placebo, thus studies that did not set a control group of chemotherapy/ placebo were not included in this meta-analysis. The following excluded studies reported similar colitis incidence
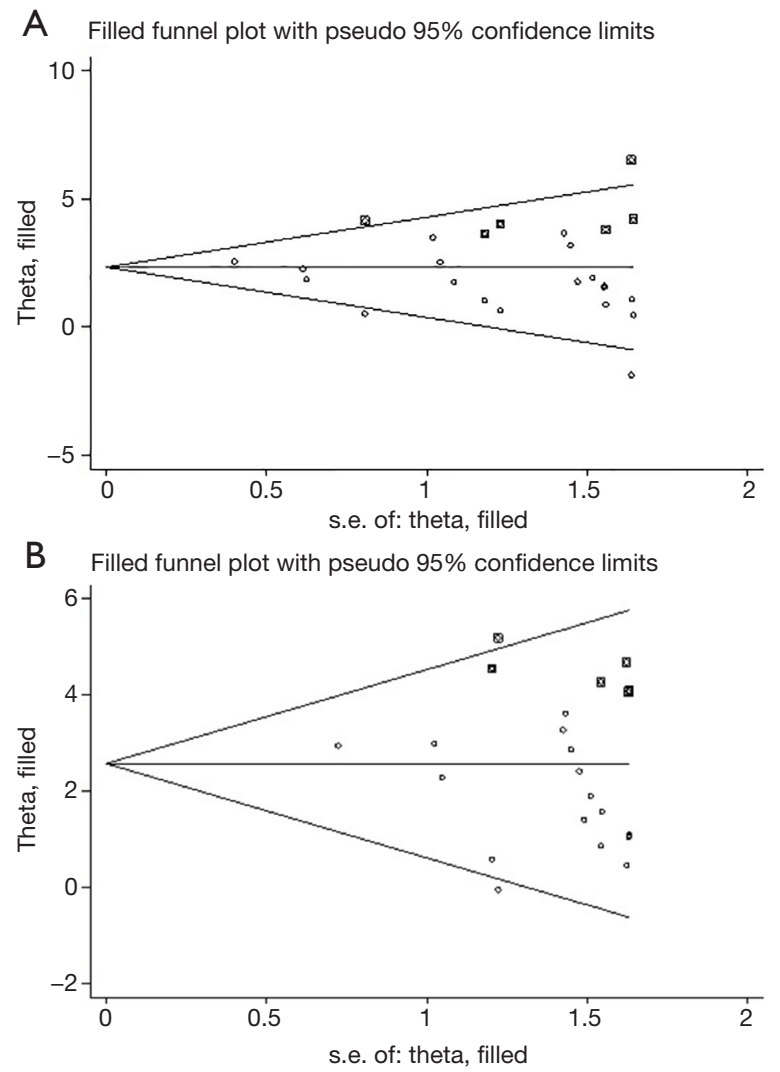

Figure 7 Trim and fill analysis. (A) Grade 1-5 colitis, (B) grade 3-5 colitis.

with the included studies in this meta-analysis. It was reported that patients with urothelial or nonurothelial urinary tract carcinoma receiving atezolizumab $1,200 \mathrm{mg}$ every 3 weeks had a risk of grade $\geq 3$ colitis at $1 \%$ (30), which falls in line with another study reporting a $1 \%$ colitis incidence rate in advanced, PD-L1-positive papillary or follicular thyroid cancer patients after receiving pembrolizumab $10 \mathrm{mg} / \mathrm{kg}$ every 2 weeks (31). A cohort of 192 patients with recurrent or metastatic head and neck squamous cell carcinoma from KEYNOTE-012, a nonrandomized multi-cohort trial, received either pembrolizumab $10 \mathrm{mg} / \mathrm{kg}$ every 2 weeks or $200 \mathrm{mg}$ every 3 weeks, of whom $0.5 \%$ patients developed immunemediated colitis (32). A 6\% colitis incidence rate was reported from a phase 2, dose-ranging study in a cohort of advanced melanoma patients on ipilimumab $10 \mathrm{mg} / \mathrm{kg}$ every 2 weeks, which is currently the standard dosage (33). Some trials evaluated the efficacy and safety of PD-1/ PD-L1 antibodies combined with CTLA-4 antibodies. A 
phase $1 \mathrm{~b}$ study applying durvalumab plus tremelimumab in NSCLC patients reported 4-24\% colitis incidence rates among groups of different dosage plans (34). Similar data were reported from a phase 2 trial applying neoadjuvant ipilimumab plus nivolumab in macroscopic stage III melanoma patients, of whom 3-24\% developed colitis during treatment (35).

Enlightened by the success of ICIs, scientists endeavor to prolong the OS even a bit further with the help of other treatment methods. ICIs combined with anti-angiogenesis therapy might very well be the best solution for solid tumors in the next decade. One study using axitinib, a VEGF receptor tyrosine-kinase inhibitor, plus pembrolizumab in patients with sarcoma reported a 3\% autoimmune colitis incidence rate (36). Another trial combining ramucirumab, a VEGF receptor-2 antagonist, with pembrolizumab reported $7 \%$ colitis events in gastric or gastro-oesophageal junction adenocarcinoma patients and $8 \%$ in urothelial carcinoma patients (37). Combination plans of ICIs with other immune treatment methods were also evaluated. Adjuvant nivolumab with vaccine in resected high-risk metastatic melanoma was reported to produce a $6.1 \%$ colitis rate (38). Autologous monocyte-derived mRNA electroporated dendritic cells (TriMixDC-MEL) plus Ipilimumab in advanced melanoma patients induced diarrhea/colitis events in $15 \%$ of the safety population (39). A meta-analysis including six trials, 445 patients, showed that combined Ipilimumab and GM-CSF produced a lower incidence of colitis versus Ipilimumab alone (combined $\mathrm{RR}=0.34,95 \% \mathrm{CI}$ : 0.13-0.86, $\mathrm{P}=0.02$ ), which was explained by the authors that GM-CSF could promote the homeostasis of the gastrointestinal tract by protecting and helping the healing of mucosa (40).

In order to more effectively recognize and manage immune related colitis, its underlying mechanism needs to be further explored. One of the mostly favorable theories is that the gut microbiome plays a vital role during this process (41). It is reported that the use of ICIs disturbs and sometimes completely breaks the homeostasis between intestinal microbiota and the mucosal immune system and such microbiome dysbiosis consequently leads to intestinal inflammation $(42,43)$. Currently acknowledged managing approach is to apply high doses of steroids, which in its mechanism would attenuate the inflammatory cascades from an over stimulated bowel immune system and restore balance in the bowel environment. If the microbiome dysbiosis theory is correct, then fecal transplantation is theoretically another solution for immune related colitis (41).
There are some limitations in this meta-analysis. According to our searching strategy, inclusion and exclusion criteria, the included twenty studies only covered melanoma, NSCLC, SCLC, prostate cancer, malignant mesothelioma, urothelial carcinoma and head-andneck squamous cell carcinoma, with the absence of other common solid tumors that positively respond to ICIs treatment, like gastric/gastroesophageal carcinoma. This could be explained by three reasons. First, many ongoing RCTs using immune therapy in other solid tumors has not yet reached endpoint, thus eventual data have not been published. Second, it is possible that certain tumor types are less likely to develop immune related colitis, consequently, studies of these tumors reported none or neglectable colitis events and were not included in this meta-analysis. Third, colitis patients could present symptoms of diarrhea, but some studies reported diarrhea events without mentioning the potential occurrence of colitis, which brings out another limitation of our study. Namely, the diagnostic standard of colitis in patients receiving ICIs is not yet universally defined. We focused on the risk of colitis as a complication from ICIs treatment and compared it with that from placebo/chemotherapy, yet some chemotherapy drugs are known to cause colitis, like irinotecan. As mentioned above in our meta-analysis, when compared with ICIs monotherapy simultaneously, chemotherapy presented higher risk of grade 1-5 and grade 3-5 colitis than placebo, suggesting that a substantial part of colitis events in the ICIs plus chemotherapy treatment arms were not caused by ICIs but by chemotherapy. Most RCTs included in our study failed to explicitly state how colitis events were recognized and how they were judged to be related to ICIs drugs or not. A phase 2 trial applying sequential administration of nivolumab and ipilimumab with a planned switch in advanced melanoma patients documented colitis events with a potential immunological cause, yet without further description of evaluation standards (44). In clinical practice, it could be rather difficult to perform differential diagnosis for a potential colitis patient, because mild cased of ICIs induced colitis could be strictly hard to tell from simple diarrhea. A phase 3 RCT comparing tremelimumab with chemotherapy in advanced melanoma patients reported $51 \%$ diarrhea/colitis in the tremelimumab arm and $18 \%$ in the chemotherapy arm, without the exact numbers of diarrhea and colitis events (45).

As ICIs begin to benefit more and more patients around the globe, a series of guidelines for ICIs induced adverse events have been established by the American Society of Clinical 
Oncology, the National Comprehensive Cancer Network, and the European Society for Medical Oncology (46). Mild cases can be managed via dose suspension, rehydration support and antidiarrheal agents, while severe cases would require systemic corticosteroid pulse treatment, sometimes even with the help of Infliximab, a TNF $\alpha$ blockade. It was reported that ICIs induced colitis patients, after first line steroid treatment, could tolerate further ICIs therapy without recurrence of symptoms if given concurrent therapy with ICIs and Infliximab (47). Overall, immune related colitis during the course of immune therapy is not hard to identify and generally controllable with timely treatment.

With reference to the currently available published RCTs, we believe that the results in this meta-analysis were reliable to some degree, and we look forward to supporting our conclusions with more multicenter, randomized controlled trials.

\section{Conclusions}

Compared with chemotherapy/placebo, ICIs combined with or without chemotherapy increase the risk of colitis in solid tumor patients. CTLA-4 antibodies produce the highest risk of colitis and PD-1 antibodies the lowest. NSCLC patients are least likely to develop colitis during ICIs treatment. Further refinements on the diagnosis and treatment protocols for ICIs induced colitis are needed.

\section{Acknowledgments}

We would like to thank Mr. Lap Kwan Chan for his help with our language issues.

Funding: None.

\section{Footnote}

Reporting Checklist: The authors have completed the PRISMA reporting checklist. Available at http://dx.doi. org/10.21037/tcr-19-2872

Conflicts of Interest: All authors have completed the ICMJE uniform disclosure form (available at http://dx.doi. org/10.21037/tcr-19-2872). The authors have no conflicts of interest to declare.

Ethical Statement: The authors are accountable for all aspects of the work in ensuring that questions related to the accuracy or integrity of any part of the work are appropriately investigated and resolved. This meta-analysis was based on previously published high quality clinical trials; thus, no ethical approval was required. Ethical approvals of each included study can be found in the original article.

Open Access Statement: This is an Open Access article distributed in accordance with the Creative Commons Attribution-NonCommercial-NoDerivs 4.0 International License (CC BY-NC-ND 4.0), which permits the noncommercial replication and distribution of the article with the strict proviso that no changes or edits are made and the original work is properly cited (including links to both the formal publication through the relevant DOI and the license). See: https://creativecommons.org/licenses/by-nc-nd/4.0/.

\section{References}

1. Topalian SL, Drake CG, Pardoll DM. Immune checkpoint blockade: a common denominator approach to cancer therapy. Cancer Cell 2015;27:450-61.

2. Postow MA, Callahan MK, Wolchok JD. Immune Checkpoint Blockade in Cancer Therapy. J Clin Oncol 2015;33:1974-82.

3. Ott PA, Hodi FS, Robert C. CTLA-4 and PD-1/PD-L1 blockade: new immunotherapeutic modalities with durable clinical benefit in melanoma patients. Clin Cancer Res 2013;19:5300-9.

4. Martins F, Sofiya L, Sykiotis GP, et al. Adverse effects of immune-checkpoint inhibitors: epidemiology, management and surveillance. Nat Rev Clin Oncol 2019;16:563-80.

5. Baxi S, Yang A, Gennarelli RL, et al. Immune-related adverse events for anti-PD-1 and anti-PD-L1 drugs: systematic review and meta-analysis. BMJ 2018;360:k793.

6. Nishijima TF, Shachar SS, Nyrop KA, et al. Safety and Tolerability of PD-1/PD-L1 Inhibitors Compared with Chemotherapy in Patients with Advanced Cancer: A MetaAnalysis. Oncologist 2017;22:470-9.

7. Bertrand A, Kostine M, Barnetche T, et al. Immune related adverse events associated with anti-CTLA-4 antibodies: systematic review and meta-analysis. BMC Med 2015;13:211.

8. Hardy T, Yin M, Chavez JA, et al. Acute fatal myocarditis after a single dose of anti-PD-1 immunotherapy, autopsy findings: a case report. Cardiovasc Pathol 2020;46:107202.

9. Sanchis-Borja M, Ricordel C, Chiappa AM, et al. Encephalitis related to immunotherapy for lung cancer: Analysis of a multicenter cohort. Lung Cancer 2020;143:36-9.

10. Hersh EM, O'Day SJ, Powderly J, et al. A phase II 
multicenter study of ipilimumab with or without dacarbazine in chemotherapy-naive patients with advanced melanoma. Invest New Drugs 2011;29:489-98.

11. Robert C, Thomas L, Bondarenko I, et al. Ipilimumab plus dacarbazine for previously untreated metastatic melanoma. N Engl J Med 2011;364:2517-26.

12. Lynch TJ, Bondarenko I, Luft A, et al. Ipilimumab in combination with paclitaxel and carboplatin as first-line treatment in stage IIIB/IV non-small-cell lung cancer: results from a randomized, double-blind, multicenter phase II study. J Clin Oncol 2012;30:2046-54.

13. Reck M, Bondarenko I, Luft A, et al. Ipilimumab in combination with paclitaxel and carboplatin as first-line therapy in extensive-disease-small-cell lung cancer: results from a randomized, double-blind, multicenter phase 2 trial. Ann Oncol 2013;24:75-83.

14. Kwon ED, Drake CG, Scher HI, et al. Ipilimumab versus placebo after radiotherapy in patients with metastatic castration-resistant prostate cancer that had progressed after docetaxel chemotherapy (CA184-043): a multicentre, randomised, double-blind, phase 3 trial. Lancet Oncol 2014;15:700-12.

15. Herbst RS, Baas P, Kim DW, et al. Pembrolizumab versus docetaxel for previously treated, PD-L1-positive, advanced non-small-cell lung cancer (KEYNOTE-010): a randomised controlled trial. Lancet 2016;387:1540-50.

16. Ribas A, Puzanov I, Dummer R, et al. Pembrolizumab versus investigator-choice chemotherapy for ipilimumabrefractory melanoma (KEYNOTE-002): a randomised, controlled, phase 2 trial. Lancet Oncol 2015;16:908-18.

17. Eggermont AM, Chiarion-Sileni V, Grob JJ, et al. Adjuvant ipilimumab versus placebo after complete resection of high-risk stage III melanoma (EORTC 18071): a randomised, double-blind, phase 3 trial. Lancet Oncol 2015;16:522-30.

18. Reck M, Luft A, Szczesna A, et al. Phase III Randomized Trial of Ipilimumab Plus Etoposide and Platinum Versus Placebo Plus Etoposide and Platinum in Extensive-Stage Small-Cell Lung Cancer. J Clin Oncol 2016;34:3740-8.

19. Rittmeyer A, Barlesi F, Waterkamp D, et al. Atezolizumab versus docetaxel in patients with previously treated non-small-cell lung cancer (OAK): a phase 3, openlabel, multicentre randomised controlled trial. Lancet 2017;389:255-65.

20. Reck M, Rodriguez-Abreu D, Robinson AG, et al. Pembrolizumab versus Chemotherapy for PD-L1Positive Non-Small-Cell Lung Cancer. N Engl J Med 2016;375:1823-33.
21. Maio M, Scherpereel A, Calabro L, et al. Tremelimumab as second-line or third-line treatment in relapsed malignant mesothelioma (DETERMINE): a multicentre, international, randomised, double-blind, placebocontrolled phase 2b trial. Lancet Oncol 2017;18:1261-73.

22. Bellmunt J, de Wit R, Vaughn DJ, et al. Pembrolizumab as Second-Line Therapy for Advanced Urothelial Carcinoma. N Engl J Med 2017;376:1015-26.

23. Cohen EEW, Soulieres D, Le Tourneau C, et al. Pembrolizumab versus methotrexate, docetaxel, or cetuximab for recurrent or metastatic head-and-neck squamous cell carcinoma (KEYNOTE-040): a randomised, open-label, phase 3 study. Lancet 2019;393:156-67.

24. Eggermont AMM, Blank CU, Mandala M, et al. Adjuvant Pembrolizumab versus Placebo in Resected Stage III Melanoma. N Engl J Med 2018;378:1789-801.

25. Brahmer J, Reckamp KL, Baas P, et al. Nivolumab versus Docetaxel in Advanced Squamous-Cell Non-Small-Cell Lung Cancer. N Engl J Med 2015;373:123-35.

26. Govindan R, Szczesna A, Ahn MJ, et al. Phase III Trial of Ipilimumab Combined With Paclitaxel and Carboplatin in Advanced Squamous Non-Small-Cell Lung Cancer. J Clin Oncol 2017;35:3449-57.

27. Robert C, Long GV, Brady B, et al. Nivolumab in previously untreated melanoma without BRAF mutation. N Engl J Med 2015;372:320-30.

28. Ferris RL, Blumenschein G, Jr., Fayette J, et al. Nivolumab for Recurrent Squamous-Cell Carcinoma of the Head and Neck. N Engl J Med 2016;375:1856-67.

29. Fehrenbacher L, Spira A, Ballinger M, et al. Atezolizumab versus docetaxel for patients with previously treated non-small-cell lung cancer (POPLAR): a multicentre, open-label, phase 2 randomised controlled trial. Lancet 2016;387:1837-46.

30. Sternberg CN, Loriot Y, James N, et al. Primary Results from SAUL, a Multinational Single-arm Safety Study of Atezolizumab Therapy for Locally Advanced or Metastatic Urothelial or Nonurothelial Carcinoma of the Urinary Tract. Eur Urol 2019;76:73-81.

31. Mehnert JM, Varga A, Brose MS, et al. Safety and antitumor activity of the anti-PD-1 antibody pembrolizumab in patients with advanced, PD-L1positive papillary or follicular thyroid cancer. BMC Cancer 2019;19:196.

32. Larkins E, Blumenthal GM, Yuan W, et al. FDA Approval Summary: Pembrolizumab for the Treatment of Recurrent or Metastatic Head and Neck Squamous Cell Carcinoma with Disease Progression on or After Platinum-Containing 
Chemotherapy. Oncologist 2017;22:873-8.

33. Wolchok JD, Neyns B, Linette G, et al. Ipilimumab monotherapy in patients with pretreated advanced melanoma: a randomised, double-blind, multicentre, phase 2, dose-ranging study. Lancet Oncol 2010;11:155-64.

34. Antonia S, Goldberg SB, Balmanoukian A, et al. Safety and antitumour activity of durvalumab plus tremelimumab in non-small cell lung cancer: a multicentre, phase $1 \mathrm{~b}$ study. Lancet Oncol 2016;17:299-308.

35. Rozeman EA, Menzies AM, van Akkooi ACJ, et al. Identification of the optimal combination dosing schedule of neoadjuvant ipilimumab plus nivolumab in macroscopic stage III melanoma (OpACIN-neo): a multicentre, phase 2, randomised, controlled trial. Lancet Oncol 2019;20:948-60.

36. Wilky BA, Trucco MM, Subhawong TK, et al. Axitinib plus pembrolizumab in patients with advanced sarcomas including alveolar soft-part sarcoma: a single-centre, single-arm, phase 2 trial. Lancet Oncol 2019;20:837-48.

37. Herbst RS, Arkenau HT, Santana-Davila R, et al. Ramucirumab plus pembrolizumab in patients with previously treated advanced non-small-cell lung cancer, gastro-oesophageal cancer, or urothelial carcinomas (JVDF): a multicohort, non-randomised, open-label, phase 1a/b trial. Lancet Oncol 2019;20:1109-23.

38. Gibney GT, Kudchadkar RR, DeConti RC, et al. Safety, correlative markers, and clinical results of adjuvant nivolumab in combination with vaccine in resected high-risk metastatic melanoma. Clin Cancer Res 2015;21:712-20.

39. Wilgenhof S, Corthals J, Heirman C, et al. Phase II Study of Autologous Monocyte-Derived mRNA Electroporated Dendritic Cells (TriMixDC-MEL) Plus Ipilimumab in Patients With Pretreated Advanced Melanoma. J Clin

Cite this article as: Wei W, Ding Y, He J, Wu J. Risk of colitis in immune checkpoint inhibitors and in chemotherapy/placebo for solid tumors: a systematic review and meta-analysis. Transl Cancer Res 2020;9(7):4173-4187. doi: 10.21037/tcr-19-2872
Oncol 2016;34:1330-8.

40. Chen P, Chen F, Zhou B. Comparisons of therapeutic efficacy and safety of ipilimumab plus GM-CSF versus ipilimumab alone in patients with cancer: a meta-analysis of outcomes. Drug Des Devel Ther 2018;12:2025-38.

41. Abu-Sbeih H, Wang Y. Gut Microbiome and Immune Checkpoint Inhibitor-Induced Enterocolitis. Dig Dis Sci 2020;65:797-9.

42. Pezo RC, Wong M, Martin A. Impact of the gut microbiota on immune checkpoint inhibitor-associated toxicities. Therap Adv Gastroenterol 2019;12:1756284819870911.

43. Carvalho FA, Koren O, Goodrich JK, et al. Transient inability to manage proteobacteria promotes chronic gut inflammation in TLR5-deficient mice. Cell Host Microbe 2012;12:139-52.

44. Weber JS, Gibney G, Sullivan RJ, et al. Sequential administration of nivolumab and ipilimumab with a planned switch in patients with advanced melanoma (CheckMate 064): an open-label, randomised, phase 2 trial. Lancet Oncol 2016;17:943-55.

45. Ribas A, Kefford R, Marshall MA, et al. Phase III randomized clinical trial comparing tremelimumab with standard-of-care chemotherapy in patients with advanced melanoma. J Clin Oncol 2013;31:616-22.

46. Haanen JB, Carbonnel F, Robert C, et al. Management of toxicities from immunotherapy: ESMO Clinical Practice Guidelines for diagnosis, treatment and follow-up. Ann Oncol 2018;29:iv264-6.

47. Johnson DH, Zobniw CM, Trinh VA, et al. Infliximab associated with faster symptom resolution compared with corticosteroids alone for the management of immunerelated enterocolitis. J Immunother Cancer 2018;6:103. 


\section{Supplementary}

A

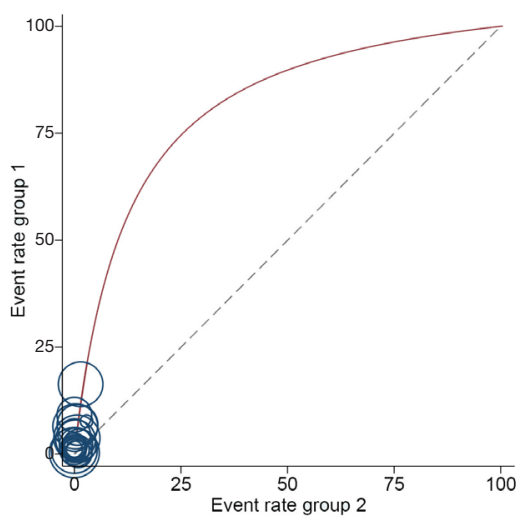

B

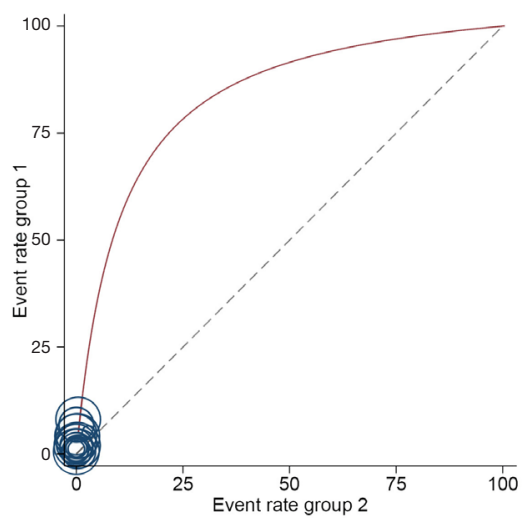

Figure S1 L'abbe plots. (A) Grade 1-5 colitis, (B) grade 3-5 colitis.

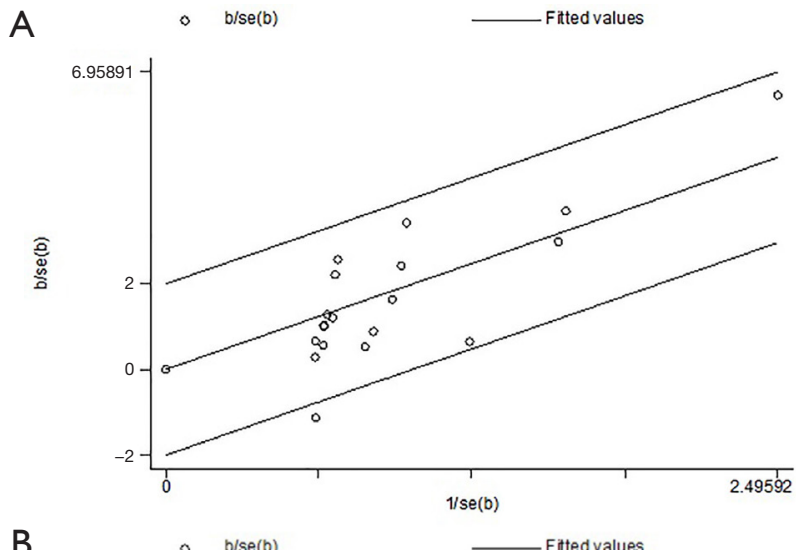

B

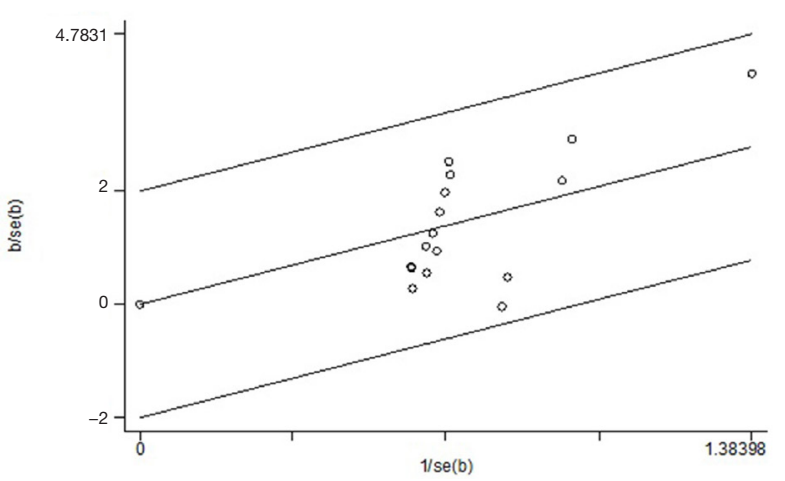

Figure S2 Galbraith radial plots. (A) Grade 1-5 colitis, (B) grade $3-5$ colitis. 

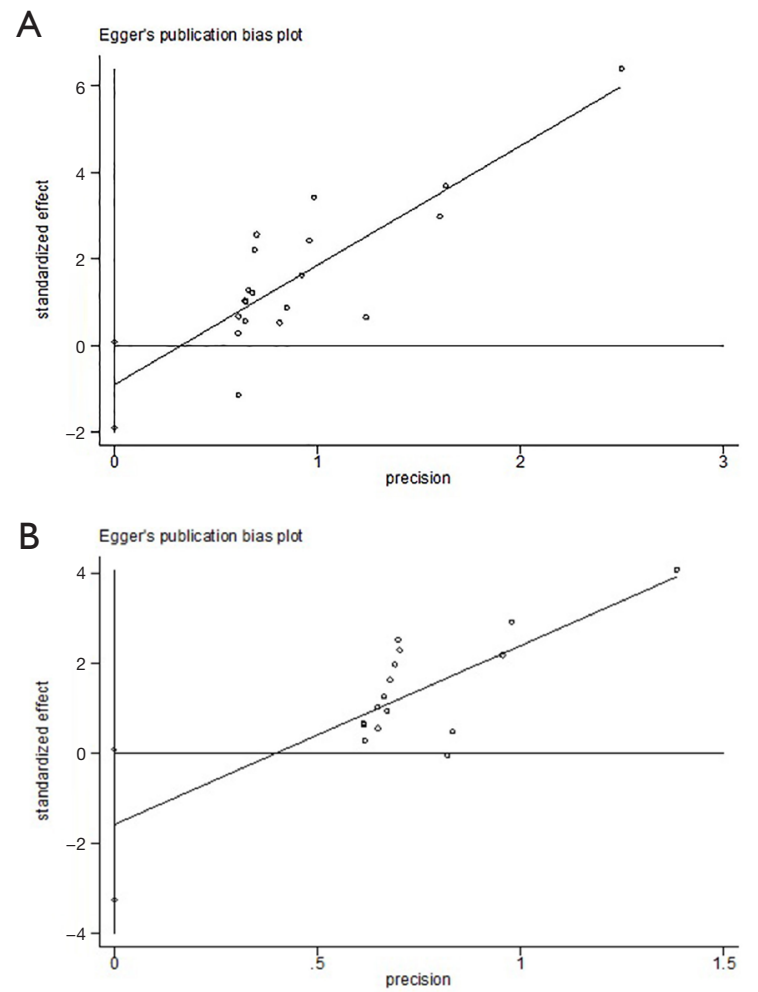

Figure S3 Egger's publication bias plots. (A) Grade 1-5 colitis, (B) grade $3-5$ colitis.

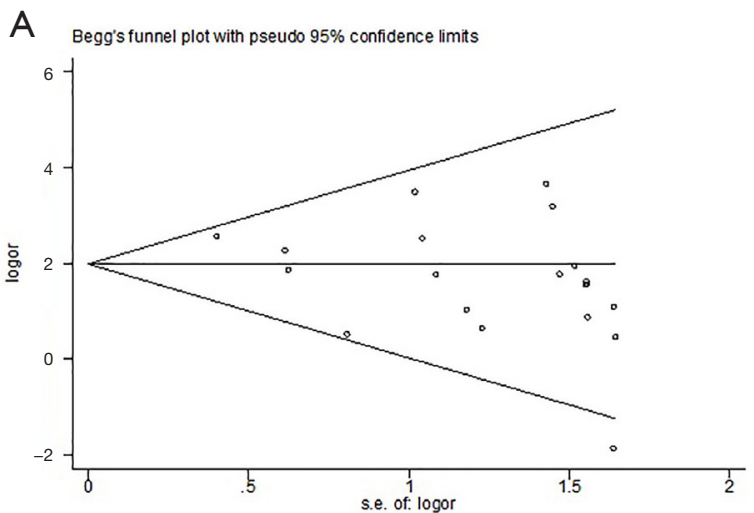

B

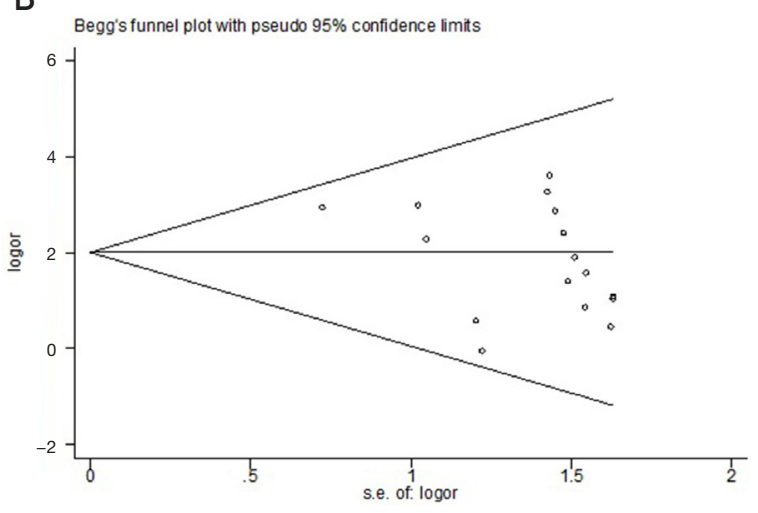

Figure S4 Begg's funnel plots. (A) Grade 1-5 colitis, (B) grade 3-5 colitis. 


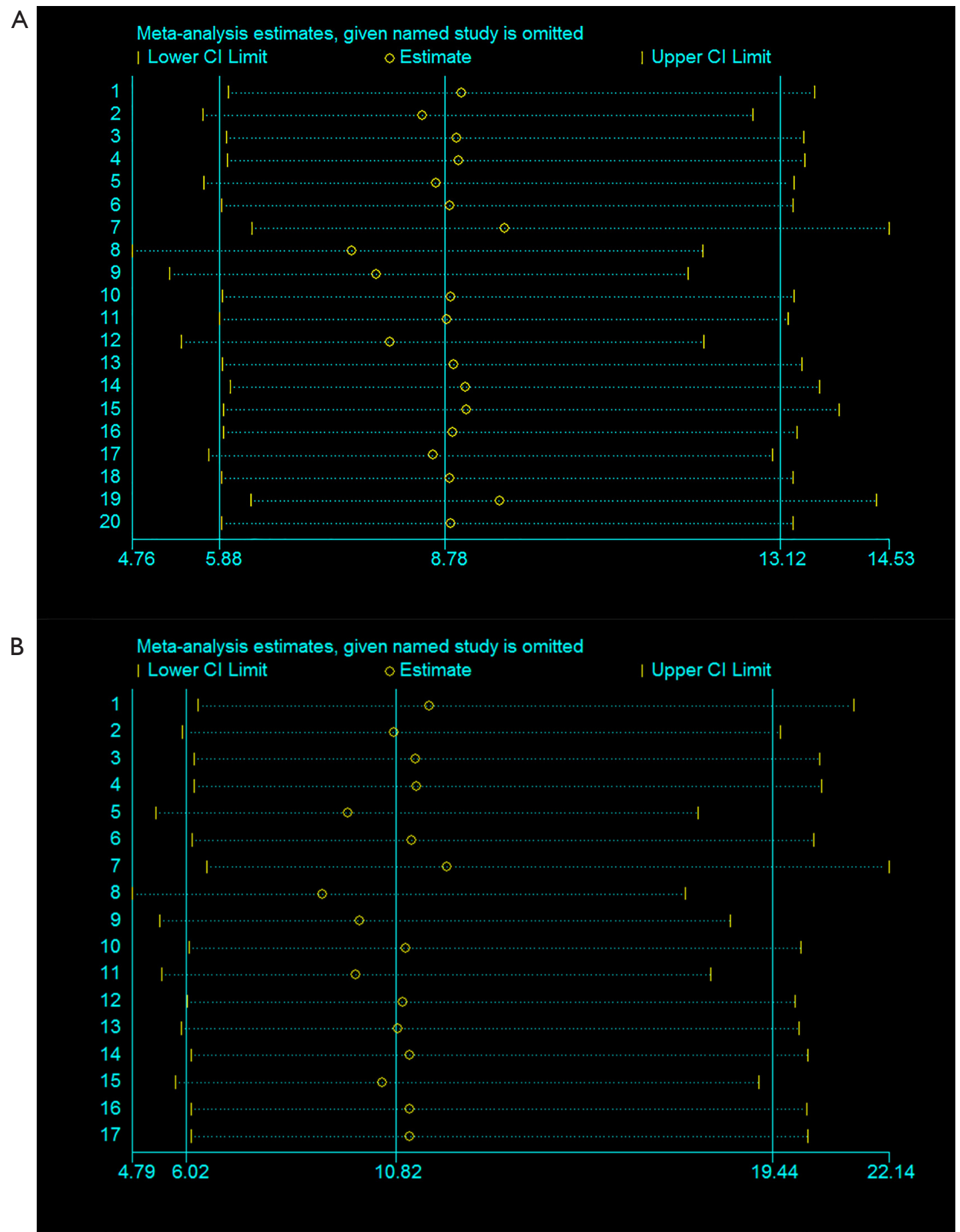

Figure S5 Influence analysis. (A) Grade 1-5 colitis, (B) grade 3-5 colitis. 